\title{
LIMITS AND BOUNDS FOR DIVIDED DIFFERENCES ON A JORDAN CURVE IN THE COMPLEX DOMAIN
}

\author{
J. H. CURTISS
}

1. Introduction. Let $S_{n+1}=\left\{z_{1}, z_{2}, \cdots, z_{n+1}\right\}$ be a set of $n+1$ complex numbers and let $f$ be a function on a set containing $S_{n+1}$ to the complex numbers. The divided difference $d_{n}=d_{n}\left(f \mid z_{1}, z_{2}, \cdots, z_{n+1}\right)$ of order $n$ formed for the function $f$ in the points ${ }^{1} S_{n+1}$ is defined in a recursive manner as follows:

$$
\begin{aligned}
d_{1} & =d_{1}\left(f \mid z_{1}, z_{2}\right)=\frac{f\left(z_{1}\right)-f\left(z_{2}\right)}{z_{1}-z_{2}} \\
d_{2} & =d_{2}\left(f \mid z_{1}, z_{2}, z_{3}\right)=\frac{d_{1}\left(f \mid z_{1}, z_{2}\right)-d_{1}\left(f \mid z_{3}, z_{2}\right)}{z_{1}-z_{3}} \\
\vdots & \\
d_{n} & =d_{n}\left(f \mid z_{1}, z_{2}, \cdots, z_{n+1}\right) \\
& =\frac{d_{n-1}\left(f \mid z_{1}, z_{2}, \cdots, z_{n}\right)-d_{n-1}\left(f \mid z_{n+1}, z_{2}, \cdots, z_{n}\right)}{z_{1}-z_{n+1}} .
\end{aligned}
$$

The definition requires further discussion when the points in $S_{n+1}$ are not all distinct. We shall suppose that they are distinct unless provision is explicitly made for coincidences.

It can be proved by induction [7, p. 15] that if

$$
\omega_{n+1}(z)=\left(z-z_{1}\right)\left(z-z_{2}\right) \cdots\left(z-z_{n+1}\right),
$$

then

$$
d_{n}=\sum_{k=1}^{n+1} \frac{f\left(z_{k}\right)}{\omega_{n+1}^{\prime}\left(z_{k}\right)},
$$

where the prime denotes differentiation of $\omega_{n+1}(z)$ with respect to $z$. This formula shows that $d_{n}$ is a symmetric function of $z_{1}, z_{2}, \cdots, z_{n+1}$.

The divided differences of a function given on the real line play a prominent role in the mathematics of computation. Their counterparts in the complex plane have appeared in various classical studies of approximation by complex polynomials. The formal algebra of complex

Received January 10, 1962. This research was supported by the United States Air Force through the Air Force Office of Scientific Research of the Air Research and Development Command, under Contract No. AF 49 (638)-862.

1 We use the words "points" and "numbers" interchangeably in referring to the arguments in divided differences. This follows the practice in interpolation theory. It is consistent within this terminology to speak of "coincident points" $z_{k}$. 
divided differences is of course much the same as for the real case, but the analytical properties of complex divided differences, such as asymptotic behavior and representability by integrals, are in some cases quite different. It would appear that these analytical properties have not received much attention in the literature, although some of them seem interesting.

A primary motivation for the present paper was the need to establish that under certain smoothness hypotheses on a function $f$ given on a Jordan curve $C$, the divided difference of $f$ of a fixed order formed in points on $C$ is uniformly bounded in modulus for all choices of the points. This property was required in a study of complex interpolation in random points [1]. The existence of the bound is proved in $\S 2$ below for the case in which $C$ is the unit circle. The extension to more general Jordan curves appears in $\S 3$. In $\S 4$, the asymptotic behavior of successive divided differences of order $n$ formed in $n+1$ points on a Jordan curve, $n=1,2, \cdots$, which in their totality become everywhere dense in a certain way on the curve, is investigated. It is found that the behavior to be expected in cases important in the theory of complex interpolation is that the $n$th divided difference multiplied by the $(n+1)$ th power of the transfinite diameter, or capacity, of the curve $C$ in question approaches the limit $\int_{C} f d z /(2 \pi i)$. Section 4 is essentially self-contained and can be read separately.

As was mentioned above, the impetus for this study came from a particular application. It is hoped that the results may turn out to be useful in other directions. However, the general spirit in which this paper is written is that of interest in the subject for itself alone, and the possible applications will not be considered further.

2. An upper bound for the modulus of a divided difference formed on the unit circle. If the numbers $z_{k}$ are all real numbers, and if $f$ is continuous on a closed interval $\bar{I}$ of the real line containing $S_{n+1}$ and possesses an $n$th derivative $f^{(n)}$ at each point of the corresponding open interval $I$, then by elementary calculus $[7$, p. 24] it can be shown that there exists a number $x_{0}$ in $I$ such that $d_{n}=f^{(n)}\left(x_{0}\right) / n$ !. Thus if $\left|f^{(n)}\right|$ is uniformly bounded everywhere on $I$, so also is $\left|d_{n}\right|$ for all choices of $S_{n+1}$ on $\bar{I}$. Again, with real points $S_{n+1}$, if $f^{(n-1)}$ is absolutely continuous [8, pp. $364 \mathrm{ff}$.$] on \bar{I}$, then the iterated integral on the right side of the following formula (in which we define $z_{n+2}$ as meaning $z_{1}$ ),

$$
\begin{aligned}
& d_{n}\left(f \mid z_{1}, z_{2}, \cdots, z_{n+1}\right) \\
& \quad=\int_{0}^{1} \int_{0}^{y_{1}} \cdots \int_{0}^{y_{n-1}} f^{(n)}\left[z_{2}+\sum_{j=1}^{n} y_{j}\left(z_{j+2}-z_{j+1}\right)\right] d y_{1} d y_{2} \cdots d y_{n},
\end{aligned}
$$

has meaning for all choices of $S_{n+1}$ in which the points $z_{j}$ are distinct, 
and indeed can be used to extend the definition of $d_{n}$ to cases involving confluent points. It is easily shown by induction that the formula is true [7, pp. 17-18]. Thus it follows with $f^{(n-1)}$ absolutely continuous and $\left|f^{(n)}\right|$, where it exists, uniformly bounded on $I$, that $\left|d_{n}\right|$ is also uniformly bounded for all choices of $S_{n+1}$ on $\bar{I}$ such that completion of the definition of $d_{n}$ through (2.1) is possible. If $M$ is the least upper bound of $\left|f^{(n)}\right|$ on $I$, then $\left|d_{n}\right| \leqq M / n$ !.

The formula (2.1) is no longer generally valid when the numbers $S_{n+1}$ are not all real, and the derivation of a bound for $\left|d_{n}\right|$ in terms of a given bound for $\left|f^{(n)}\right|$ is not so readily accomplished. In the remainder of the section we shall consider this problem in the case in which $S_{n+1}$ lies on the unit circle in the complex plane.

In the development, we shall use a complex-variable type of interpretation of the derivatives of a function $g$ given on the circle $C:|z|=1$. The symbol $g^{(1)}\left(z_{1}\right)$ will mean

$$
\lim _{z \rightarrow z_{1}} d_{1}\left(g \mid z, z_{1}\right)=\lim _{z \rightarrow z_{1}} \frac{g(z)-g\left(z_{1}\right)}{z-z_{1}},|z|=1,\left|z_{1}\right|=1,
$$

provided of course that the limit exists. Higher derivatives $g^{(k)}$ are to be defined recursively. The circle $C$ can be parametrized in a one-toone manner by the equation $z=e^{i \theta}$, with $\alpha \leqq \theta<\alpha+2 \pi$, where $\alpha$ is chosen arbitrarily. If this is done, then

$$
g^{(1)}(z)=\frac{d g}{d \theta} \cdot \frac{d \theta}{d e^{i \theta}}=\frac{d g}{d \theta} \cdot \frac{1}{i e^{i \theta}} .
$$

The chief result is this:

THEOREM 2.1 Let the function $f$ be given on $C:|z|=1$ together with its first $n-1$ derivatives $f^{(1)}, f^{(2)}, \cdots, f^{(n-1)}$. Let the points $S_{n+1}$ lie on $C$ and be distinct. Then if $f^{(n-1)}$ satisfies the Lipschitz condition:

$$
\left|f^{(n-1)}(z)-f^{(n-1)}(t)\right| \leqq \lambda|z-t|, \lambda>0,
$$

for all $z$ and $t$ on $C$, it follows that

$$
\text { (2.2) }\left|d_{n}\left(f \mid z_{1}, z_{2}, \cdots, z_{n+1}\right)\right| \leqq \frac{\frac{\pi}{2}\left(\frac{\sqrt{\pi}}{2}\right)^{n-1} \lambda_{n}}{\Gamma\left(\frac{n+1}{2}\right)}
$$

uniformly for all such $S_{n+1}$, where $\lambda_{n}$ is the least upper bound of

$$
\left|f^{(n)}(z)\right|,|z|=1 \text {. }
$$

The symbol $\Gamma$ in (2.2) refers to the Gamma Function. 
The hypothesis on $f^{(n-1)}$ has the implication that $f^{(n-1)}$ be absolutely continuous on $|z|=1$, and that it therefore be the indefinite integral of a derivative $f^{(n)}$ existing everywhere on $|z|=1$ with the possible exception of a set of Lebesgue measure zero. Moreover the implication is that $\left|f^{(n)}\right|$, on the set where it exists, is bounded and its least upper bound $\lambda_{n}$ does not exceed $\lambda$.

Our proof involves integral representations, and it is important to be explicit about the integral calculus to be used.

Consider two points $e^{i \alpha_{2}}$ and $e^{i \alpha_{1}}$ on the unit circle. The complex line integral of a function $g$ given on the unit circle extended over either one of the two arcs of the circle joining these points, directed from $e^{i \alpha_{2}}$ to $e^{i \alpha_{1}}$, is to be defined as a Lebesgue integral with respect to the parameter $\theta$ in the parametrization $z=e^{i \theta}$. That is, if $A$ is the chosen directed arc, then

$$
\int_{A} g(z) d z=\int_{\alpha_{2}}^{\alpha_{1}} g\left(e^{i \theta}\right) i e^{i \theta} d \theta .
$$

If $g$ is continuous in a neighborhood of $e^{i \alpha_{1}}$, then

$$
\frac{d}{d e^{i \alpha_{1}}} \int_{A} g(z) d z=\frac{g\left(e^{i \alpha_{1}}\right) i e^{i \alpha_{1}}}{i e^{i \alpha_{1}}}=g\left(e^{i \alpha_{1}}\right) .
$$

The notation for the integral on the right side of (2.3) is ambiguous in that it does not indicate which one of the two possible directed arcs $A$ is being integrated over. However in the sequel we shall be dealing only with complex line integrals on $|z|=1$ which are independent of the path of integration. Such an integral extended over either arc directed from $z_{2}$ to $z_{1},\left|z_{1}\right|=\left|z_{2}\right|=1$, will be denoted by

$$
\int_{z_{2}}^{z_{1}} g(z) d z
$$

If the two arcs joining $z_{2}$ to $z_{1}$ are are of equal length, then $\alpha_{2}=\alpha_{1} \pm \pi$, so the variation of $\theta$ in (2.3) is over a closed interval of length $\pi$ of which one endpoint is $\alpha_{2}$. If the two paths are not of equal length, then the shorter one corresponds under $z=e^{i \theta}$ to an interval of values of $\theta$ of which one endpoint is $\alpha_{2}$ and the other one, say $\alpha^{\prime}$, is such that $z_{1}=e^{i \alpha_{1}}=e^{i \alpha^{\prime}}$ and $\left|\alpha_{2}-\alpha^{\prime}\right|<\pi$. (For example, if $\alpha_{1}$ and $\alpha_{2}$ are restricted to the interval $[0,2 \pi]$ and if $\alpha_{2}>\alpha_{1}, \alpha_{2}-\alpha_{1}>\pi$, then we take $\alpha^{\prime}=2 \pi+\alpha_{1}$.)

We shall now drop the parentheses around superscripts indicating derivatives of functions, but it is to be understood that superscripts can also be exponents when the context requires, as in $\left(z-z_{2}\right)^{b}$. In the case of divided differences, a derivative superscript will always indicate a partial derivative with respect to the first argument when the 
LIMITS AND BOUNDS FOR DIVIDED DIFFERENCES ON A JORDAN CURVE 1221 notation in the first paragraph of the Introduction is being used. That is

$$
d_{m}^{k}=d_{m}^{k}\left(f \mid z_{1}, z_{2}, \cdots, z_{m+1}\right) \frac{\partial^{(k)} d_{m}\left(f \mid z_{1}, z_{2}, \cdots, z_{m+1}\right)}{\partial z_{1}^{(k)}}
$$

To prove Theorem 2.1 we need two lemmas, of which the first is as follows.

LEMmA 2.1 Let the function $f$ given on $C:|z|=1$ be such that its $(n-1)$ st derivative exists everywhere on $C$ and is absolutely continuous. Then

$$
\begin{aligned}
& d_{1}^{h-1}\left(f \mid z_{1}, z_{2}\right)=\frac{\int_{z_{2}}^{z_{1}}\left(t-z_{2}\right)^{n-1} f^{h}(t) d t}{\left(z_{1}-z_{2}\right)^{h}}, \\
& \left|z_{1}\right|=1, \quad\left|z_{2}\right|=1, \quad z_{1} \neq z_{2}, \quad h=1,2, \cdots, n .
\end{aligned}
$$

The integral is independent of the path of integration on $C$.

The absolute continuity of $f^{n-1}$ implies the absolute continuity of $f, f^{2}, \cdots, f^{n-2}$, and so implies that each of these functions including $f^{n-1}$ is the indefinite integral of its derivative.

In the case $h=1$, with $z_{1}=e^{i \alpha_{1}}, z_{2}=e^{i \alpha_{2}}$,

$$
\begin{gathered}
\frac{\int_{z_{2}}^{z_{1}} f^{\prime}(t) d t}{z_{1}-z_{2}}=\frac{\int_{\alpha_{2}}^{\alpha_{1}} f^{\prime}\left(e^{i \theta}\right) i e^{i \theta} d \theta}{z_{1}-z_{2}} \\
=\frac{f\left(z_{1}\right)-f\left(z_{2}\right)}{z_{1}-z_{2}} \\
=\frac{\int_{a_{2}}^{a_{1} \pm 2 \pi} f^{\prime}\left(e^{i \theta}\right) i e^{i \theta} d \theta}{z_{1}-z_{2}} .
\end{gathered}
$$

The second and fourth members of the equation show that whether $\alpha_{1}>\alpha_{2}$ or $\alpha_{1}<\alpha_{2}$, the integral is independent of the path. Thus the Lemma is true for $h=1$.

Suppose now that (2.5) gives a valid representation of $d_{1}^{k-1}, 1 \leqq k<h$, with the integral independent of the path and $z_{1} \neq z_{2}$. Then using (2.4), we have after a brief computation

$$
d_{1}^{k}=\frac{\partial d_{1}^{k-1}}{\partial z_{1}} \frac{\left(z_{1}-z_{2}\right)^{k} f^{k}\left(z_{1}\right)-k \int_{z_{2}}^{z_{1}}\left(t-z_{2}\right)^{k-1} f^{k}(t) d t}{\left(z_{1}-z_{2}\right)^{k+1}} .
$$

Because of the absolute continuity of $f^{k}$, integration by parts is valid 
in the integral in (2.6), with $f^{k}$ to be differentiated and $\left(t-z_{2}\right)^{k-1}$ to be integrated with respect to $t$. We thereby immediately obtain (2.5) with $h=k+1$, and the integral is again independent of the path. The premises of the induction are true for $k=1$, so this establishes the Lemma.

Lemma 2.2 Let the function $g$ be given at all points on $|z|=1$ with the possible exception of a set of Lebesgue measure zero; let $|g(z)| \leqq M$ where defined on $|z|=1$, and let $g$ be such that

$$
I_{k}\left(z_{1}, z_{2}\right)=\int_{z_{2}}^{z_{1}}\left(t-z_{2}\right)^{k} g(t) d t, \quad\left|z_{1}\right|=\left|z_{2}\right|=1, \quad k \geqq 0,
$$

is independent of the path of integration. Then

$$
\left|\frac{I_{k}\left(z_{1}, z_{2}\right)}{\left(z_{1}-z_{2}\right)^{k+1}}\right| \leqq \frac{\sqrt{\pi}}{2} \cdot \frac{\Gamma\left(\frac{k+1}{2}\right)}{\Gamma\left(\frac{k+2}{2}\right)} M, k=0,1,2, \cdots,
$$

for all $z_{1}$ and $z_{2}, z_{1} \neq z_{2}$, on $|z|=1$.

When $k=0$, the right side of (2.7) reduces to $\pi M / 2$.

For the proof, we make the shorter arc joining $z_{2}=e^{i \alpha_{2}}$ and $z_{1}=$ $e^{i \alpha_{1}}$ (or either of the two ares if they are equal in length) correspond under $z=e^{i \theta}$ to a $\theta$-interval $\left[\alpha_{2}, \alpha^{\prime}\right]$ or $\left[\alpha^{\prime}, \alpha_{2}\right]$, where $\alpha^{\prime}$ is such that $z_{1}=e^{i \alpha^{\prime}}$ and $\left|\alpha_{2}-\alpha^{\prime}\right| \leqq \pi$. Thus

$$
I_{k}\left(z_{1}, z_{2}\right)=\int_{\alpha_{2}}^{\alpha^{\prime}}\left(e^{i \theta}-e^{i \alpha_{2}}\right)^{k} g\left(e^{i \theta}\right) i e^{i \theta} d \theta .
$$

For the case $k=0$, we use the inequality $|\sin \theta| \geqq|2 \theta| \pi \mid,-\pi / 2 \leqq \theta \leqq$ $\pi / 2$, which is merely an expression of the fact that $\sin \theta$ is convex on $[0, \pi / 2]$. We also use the identity $e^{i \alpha}-e^{i \beta}=2 i e^{i(\alpha+\beta) / 2} \sin [(\alpha-\beta) / 2]$. Then since $\left|\alpha^{\prime}-\alpha_{2}\right| / 2 \leqq \pi / 2$, it follows that $\left|z_{1}-z_{2}\right|=2 \sin \left[\left(\alpha^{\prime}-\alpha_{2}\right) / 2\right] \mid \geqq$ $(2 / \pi)\left|2\left(\alpha^{\prime}-\alpha_{2}\right) / 2\right|$. Now $\left|I_{0}\left(z_{1}, z_{2}\right)\right| \leqq M\left|\alpha^{\prime}-\alpha_{2}\right|$, so the inequality (2.7) follows at once for $k=0$.

For $k \geqq 1$ we have (recalling the restriction on $\left|\alpha^{\prime}-\alpha_{2}\right|$ ),

$$
\begin{aligned}
\frac{\left|I_{k}\left(z_{1}, z_{2}\right)\right|}{\left|z_{1}-z_{2}\right|^{k+1}} & \leqq \frac{M 2^{k}\left|\int_{\alpha_{2}}^{\alpha^{\prime}}\right| \sin ^{k}\left(\frac{\theta-\alpha_{2}}{2}\right)|d \theta|}{2^{k+1}\left|\sin ^{k+1} \frac{\alpha^{\prime}-\alpha_{2}}{2}\right|} \\
& =\frac{M}{2} \frac{\left|\int_{\alpha_{2}}^{\alpha_{1}} \sin ^{k}\right| \frac{\theta-\alpha_{2}}{2}|d \theta|}{\sin ^{k+1}\left|\frac{\alpha^{\prime}-\alpha_{2}}{2}\right|} .
\end{aligned}
$$


We make the substitution $\beta=\left|\theta-\alpha_{2}\right| / 2$ in the integral and let $\gamma=$ $\left|\alpha^{\prime}-\alpha_{2}\right| / 2 \leqq \pi / 2$. By examination of the various cases corresponding to $k$ even or odd and $\alpha^{\prime}<\alpha_{2}, \alpha^{\prime}>\alpha_{2}$ we find that the righthand member of (2.8) is always equal to

$$
M \int_{0}^{\gamma} \frac{\sin ^{k} \beta d \beta}{\sin ^{k+1} \gamma}=M S(\gamma) .
$$

Inspection of its derivative shows that $S(\gamma)$ increases steadily with $\gamma$ on the interval $0 \leqq \gamma \leqq \pi / 2$. The value of $S(\pi / 2)$ is given by the well known formula

$$
\int_{0}^{\pi / 2} \sin ^{k} \theta d \theta=\frac{\sqrt{\pi}}{2} \frac{\Gamma\left(\frac{k+1}{2}\right) M}{\Gamma\left(\frac{k+2}{2}\right)}
$$

Thus

$$
\frac{\left|I_{k}\left(z_{1}, z_{2}\right)\right|}{\left|z_{1}-z_{2}\right|^{k+1}} \leqq M S\left(\frac{\pi}{2}\right)=\frac{\sqrt{2}}{2} \frac{\Gamma\left(\frac{k+1}{2}\right)}{\Gamma\left(\frac{k+2}{2}\right)} M,
$$

as was to be proved.

Now let $g$ in Lemma 2.2 be $f^{n}$ and $M$ be $\lambda_{n}$, where $f$ is the function appearing in the Theorem. The two lemmas establish that

$$
d_{1}^{n-1}\left(f \mid z_{1}, z_{2}\right)=\frac{\lambda_{n} \sqrt{\pi} \Gamma^{r}\left(\frac{n}{2}\right)}{2 \Gamma\left(\frac{n+1}{2}\right)}, \quad z_{1} \neq z_{2} .
$$

As a function of $z_{1}, d_{1}^{n-1}$ is continuous for $z_{1} \neq z_{2}$ and uniformly bounded in modulus. Consider next $d_{1}^{n-2}\left(f z_{1}, z_{2}\right)$ as a function of $z_{1}$. This function has a continuous derivative for $z_{1} \neq z_{2}$, which is moreover uniformly bounded in modulus. Therefore this function is absolutely continuous in $z_{1}$ for $z_{1}$ on any closed arc of the unit circle not containing $z_{2}$. But the uniform boundedness of $\left|d_{1}^{n-1}\right|$ implies that $d_{1}^{n-2}\left(f \mid z_{1}, z_{2}\right)$ is of uniformly bounded variation in $z_{1}$ on the entire unit circle with the point $z_{2}$ deleted. By a well-known theorem [8, p. 372, Ex. 6] it follows that as $z_{1}$ approaches $z_{2}$ from either side, $d_{1}^{n-2}$ approaches a limit; and if the limit is the same for approach from either side, then when the definition of $d_{1}^{n-2}$ is completed by this limit at $z_{1}=z_{2}$ the function $d_{1}^{n-2}$ will be an absolutely continuous function for all $z_{1}$ on $\left|z_{1}\right|=1$. To investigate the limit, we write (2.5) in the form 


$$
d_{1}^{n-2}=\left[\frac{\int_{\alpha_{2}}^{\alpha_{1}}\left(e^{i \theta}-e^{i \alpha_{2}}\right)^{n-2} f^{n-1}\left(e^{i \theta}\right) i e^{i \theta} d \theta}{\left(\sin \frac{\alpha_{1}-\alpha_{2}}{2}\right)^{n-1}}\right] \cdot \frac{1}{\left(2 i e^{i\left(\alpha_{1}+\alpha_{2}\right) / 2}\right)^{n-1}} .
$$

The limit as $z_{1} \rightarrow z_{2}, \alpha_{1} \rightarrow \alpha_{2}$, of the expression in square brackets can be evaluated by l'Hospital's rule used with (2.4) and with the fact that $f^{n-1}$ is continuous. We find that for $z_{1}$ approaching $z_{2}$ on either side, there is the unique limit

$$
\lim _{z_{1} \rightarrow z_{2}} d_{1}^{n-2}\left(f \mid z_{1}, z_{2}\right)=\frac{f^{n-1}\left(z_{2}\right)}{n-1} .
$$

Thus with proper completion of the definition of $d_{1}^{n-2}$ at $z_{1}=z_{2}$, this function is an absolutely continuous function of $z_{1}$ on $\left|z_{1}\right|=1$. Similarly we can complete the definition of $d_{1}^{h}\left(f \mid z_{1}, z_{2}\right)$ for $h=n-3, n-4, \cdots$, 1,0 , so that the resulting function is in each case an absolutely continuous function of $z_{1}$ on $\left|z_{1}\right|=1$. We assume henceforth without change in notation that for each relevant value of $h$, the proper extension of the definition of $d_{1}^{h}$ at $z_{1}=z_{2}$ has been made.

What this establishes is that the completed first order divided difference $d_{1}\left(f \mid z_{1}, z_{2}\right)$, as a function of $z_{1}$, together with its first $n-2$ partial derivatives with respect to $z_{1}$, have the same smoothness and integrability properties as does $f$ and its first $n-1$ derivatives. That is to say, $d_{1}, d_{1}^{2}, \cdots, d_{1}^{n-2}$ are absolutely continuous functions of $z_{1}$ and moreover the derivative of $d_{1}^{n-2}$, where it exists, is uniformly bounded in modulus.

The absolute continuity of the derivatives permits the inductive argument which we used to establish (2.5) to be used again to prove that

$$
\begin{aligned}
& d_{2}^{h-1}\left(f \mid z_{1}, z_{2}, z_{3}\right)=\frac{\partial^{h-1}}{\partial z_{1}^{h-1}} d_{1}\left(d_{1}\left(f \mid z, z_{2}\right) \mid z_{1}, z_{3}\right) \\
& =\frac{\int_{z_{3}}^{z_{1}}\left(t-z_{3}\right)^{h-1} d_{1}^{h}\left(f \mid t, z_{2}\right) d t}{\left(z_{1}-z_{3}\right)^{h}} \\
& \quad\left|z_{1}\right|=1,\left|z_{2}\right|=1, z_{1} \neq z_{3}, h=1,2, \cdots, n-1 .
\end{aligned}
$$

By (2.9) and Lemma 2.2, $d_{2}^{n-2}$ as a function of $z_{1}$ is uniformly bounded in modulus. (It is not important at the moment to know how the bound depends on $z_{2}$ and $z_{3}$.) The definitions of $d_{2}^{n-3}, d_{2}^{n-4}, \cdots, d_{2}$ can now be completed by continuity at $z_{1}=z_{3}$ so that in each case the resulting function of $z_{1}$ is absolutely continuous on $\left|z_{1}\right|=1$. Again we assume without change of notation that the proper extensions have been made. 
Proceeding in this way, we establish the chain of equations

$$
d_{k}^{n-k}=\frac{\int_{z_{k+1}}^{z_{1}}\left(t-z_{k+1}\right)^{n-k} d_{k-1}^{n-k+1}\left(f \mid t, z_{2}, \cdots, z_{k}\right) d t}{\left(z_{1}-z_{k+1}\right)^{n-k+1}} \quad, \quad k=1,2, \cdots, n,
$$

in which $d_{n}^{0}=d_{n}, d_{0}^{n}=f^{n}$. Theorem 2.1 now can be proved by back substitution into (2.10), beginning with (2.9) and using Lemma 2.2 at each stage. Thus to start with, at least for $z_{1} \neq z_{2}$ and $z_{1} \neq z_{3}$,

$$
\left|d_{2}^{n-2}\right| \leqq \frac{\sqrt{\pi}}{2} \frac{\Gamma\left(\frac{n-1}{2}\right)}{\Gamma\left(\frac{n}{2}\right)}\left[\frac{\lambda_{n} \sqrt{\pi} \Gamma\left(\frac{n}{2}\right)}{2 \Gamma\left(\frac{n+1}{2}\right)}\right] .
$$

Similarly,

$$
\begin{aligned}
\left|d_{3}^{n-3}\right| & \leqq\left(\frac{\sqrt{\pi} \Gamma\left(\frac{n-2}{2}\right)}{2 \Gamma\left(\frac{n-1}{2}\right)}\right)\left(\frac{\sqrt{\pi} \Gamma\left(\frac{n-1}{2}\right)}{2 \Gamma\left(\frac{n}{2}\right)}\right)\left[\frac{\lambda_{n} \sqrt{\pi}}{2} \frac{\Gamma\left(\frac{n}{2}\right)}{\Gamma\left(\frac{n+1}{2}\right)}\right] \\
& =\left(\frac{\sqrt{\pi}}{2}\right)^{3} \frac{\Gamma\left(\frac{n-2}{2}\right)}{\Gamma\left(\frac{n+1}{2}\right)} \lambda_{n},
\end{aligned}
$$

and so forth. We finally find that

$$
\left|d_{n}^{0}\right| \leqq \frac{\pi}{2}\left(\frac{\sqrt{\pi}}{2}\right)^{n-1} \frac{\lambda_{n}}{\Gamma\left(\frac{n+1}{2}\right)},
$$

as stated in the conclusion of the Theorem.

It is clear from the proof that under the hypotheses of Theorem 2.1 on $f$, it is possible to extend the definition of $d_{n}$ by continuity so as to admit point sets $S_{n+1}$ in which coincidences occur, and then (2.2) will still be valid. However we shall not study this question in detail under the hypotheses of Theorem 2.1 on $f$, which were chosen as being natural to achieve boundedness of $\left|d_{n}\right|$ in the case of distinct points. (The boundedness of $d_{1}=\left|f\left(z_{1}\right)-f\left(z_{2}\right)\right| /\left|z_{1}-z_{2}\right|$ is equivalent to a Lipschitz condition on $f$.)

The method of proof with only slight modifications can be used to establish the following result:

Theorem 2.2 Under the hypothesis of Theorem 2.1 concerning $f$, 
and with the added hypothesis that $f^{(n)}$ is continuous on some open arc of $|z|=1$ containing the point $z_{1}$, the following equation completes the definition of $d_{n}$ by continuity for the case in which all the points $S_{n+1}$ coincide at $z_{1}$ :

$$
d_{n}\left(f \mid z_{1}, z_{1}, \cdots, z_{1}\right)=\frac{f^{(n)}\left(z_{1}\right)}{n !} .
$$

If $f^{(n)}$ is everywhere continuous on $|z|=1$, then (2.2) is valid after proper completion of the definition of $d_{n}$ for all choices of $S_{n+1}$ without restrictions as to coincidences.

We conclude this section with two comments. In the first place it is clear that by repeated back-substitution into (2.10), a single formula for $d_{n}$ in terms of $f^{(n)}$ involving repeated integration can be written out. It would be somewhat similar in appearance to a variant of (2.1) which appears in [5, p. 18, ex. 7].

In the second place, it may be that for $n \geqq 2$ the bound in (2.2) can be improved. For $n=1$ it is the best bound possible, as can be seen from this trivial example: Let $f$ be real and let its graph over a period in the $\left(\theta, f\left(e^{i \theta}\right)\right)$-plane be a line segment joining $(0,0)$ to $(\pi, \pi)$ and another line segment joining $(\pi, \pi)$ to $(2 \pi, 0)$. For this function the maximum of $d_{1}$ is $\pi / 2$, the least upper bound of $\left|f^{\prime}\right|$ is one, and the right side of $(2.2)$ is $(\pi / 2) \cdot 1$, which is as small as it can be. However the general bound was derived through Lemma 2.2 in which the two points $z_{1}$ and $z_{2}$ were placed at opposite ends of a diameter to obtain the numerical appraisal. Such wide-apart spacing is of course not possible for the case of three or more points on the unit circle. The bound given by (2.1) in the real case under the hypothesis of Theorem 2.1 is $\lambda_{n} / n$ !, which is much smaller than that in (2.2).

3. Boundedness of the modulus of a divided difference formed on a general Jordan curve. A generalization of Theorem 2.1 to the case in which the unit circle is replaced by a more general Jordan curve is not hard to derive. In doing so, for simplicity we shall not try to keep track of the structure of the upper bound, and shall suppress various details in the proof.

A Jordan curve is homeomorphic to a circle. It can be represented by a parametric equation $z=\phi(\theta)$, where $\phi$ is continuous in the real variable $\theta$ with period $2 \pi$, and where for each given point $z$ on the curve, any two solutions of $z=\phi(\theta)$ differ by an integral multiple of $2 \pi$. Our considerations here will be restricted to Jordan curves such that the first derivative $d \phi / b \theta=\psi(\theta)$ exists for all $\theta$ and is continuous, and $\psi(\theta) \neq 0$ for all $\theta$. Such a Jordan curve will be said to be "admissible". (Presumably in what follows the definition of admissibility 
can be slightly relaxed.)

LEMMA 3.1. If $z=\phi(\theta)$ is a parametric equation of an admissible Jordan curve, then there exist numbers $m$ and $M, 0<m<M$, such that

$$
m \leqq\left|\frac{\phi\left(\theta_{1}\right)-\phi\left(\theta_{2}\right)}{e^{i \theta_{1}}-e^{i \theta_{2}}}\right| \leqq M
$$

for all $\theta_{1}$ and $\theta_{2}$.

The divided difference appearing in the above inequality is to be interpreted as meaning $\psi\left(\theta_{2}\right) / i e^{i \theta_{2}}$ when $\theta_{1}=\theta_{2}$.

The existence of an upper bound $M$ follows from Lemma 2.2 with $k=0$ and $g\left(e^{i \theta}\right)=\psi(\theta) / i e^{i \theta}$. The existence of the lower bound can be established by an elementary indirect argument which we omit ${ }^{2}$.

As in the unit circle case, it is convenient to interpret the derivatives of a function on a Jordan curve to the complex numbers as limits of complex-variable difference quotients. Specifically for any function $g$ given on a Jordan curve $C$, the symbol $g^{\prime}\left(z_{1}\right)$ means $\lim _{z \rightarrow z_{1}} d_{1}\left(g \mid z, z_{1}\right)$, $z$ and $z_{1}$ on $C$, and higher derivatives are to be defined recursively. If $C$ is admissible, then

$$
g^{\prime}(z)=\frac{d(g(\phi(\theta)))}{d \theta} \cdot \frac{1}{\psi(\theta)}
$$

Integrals are to be defined as in (2.3) with $i e^{i \theta}$ in that formula replaced by $\psi(\theta)$. With this replacement and with $e^{i \alpha_{1}}$ replaced by $\phi\left(\alpha_{1}\right),(2.4)$ is is valid. An integral over $C$ with limits of integration $z_{1}$ and $z_{2}$ which is independent of the path will be written as

$$
\int_{z_{2}}^{z_{1}} g(z) d z
$$

The notation implies of course that the arc over which the integration takes place is directed from $z_{2}$ to $z_{1}$. The derivatives of divided differences are always partials with respect to the first apparent argument.

The generalization of Theorem 2.1 is as follows:

THEOREM 3.1. Let the function $f$ be given on an admissible Jordan curve $C$, together with $f^{1}, f^{2}, \cdots, f^{n-1}$. Let $f^{n-1}$ satisfy the Lipschitz condition

$$
\left|f^{n-1}(z)-f^{n-1}(t)\right| \leqq \lambda|z-t|, \lambda>0,
$$

for all $z$ and $t$ on $C$. Let the points $S_{n+1}=\left\{z_{1}, z_{2}, \cdots, z_{n+1}\right\}$ lie on $C$ and be distinct. Then there exists a constant $M$ depending only on $n, \lambda$, and $C$, and independent of $S_{n+1}$, such that $\left|d_{n}\left(f \mid z_{1}, z_{2}, \cdots, z_{n+1}\right)\right| \leqq M$.

${ }^{2}$ Various related but deeper results may be found in [6, Section 2.5]. 
The proof starts with a generalization of Lemma 2.1.

Lemma 3.2. Let the function $f$, given on an admissible Jordan curve $C$, be such that its $(n-1)$ th derivative exists everywhere on $C$ and is absolutely continuous as a function of $\theta, z=\phi(\theta)$. Then

$$
d_{1}^{h-1}\left(f \mid z_{1}, z_{2}\right)=\frac{\int_{z_{2}}^{z_{1}}\left(t-z_{2}\right)^{h-1} f^{h}(t) d t}{\left(z_{1}-z_{2}\right)^{h}},
$$

for $z_{1}$ and $z_{2}$ on $C, z_{1} \neq z_{2}, h=1,2, \cdots, n$, and the integral is independent of the path of integration on $C$.

The argument used to establish Lemma 2.1 carries over to Lemma 3.2 with only minor changes, and will not be restated here.

Lemma 3.3. Let the function $G$ be given on the admissible Jordan curve $C$ with the possible exception of a set of Lebesgue measure zero; let $|G|$ be bounded on $C$ and be such that

$$
J_{k}\left(z_{1}, z_{2}\right)=\int_{z_{2}}^{z_{1}}\left(t-z_{2}\right)^{k} G(t) d t
$$

is independent of the path of integration on $C$ for all $z_{1}$ and $z_{2}$ on $C$. Then for each $k, k=0,1, \cdots$, there exists a constant $M_{k}$, depending only on $G$ and $C$, and such that

$$
\left|\frac{J_{k}\left(z_{1}, z_{2}\right)}{\left(z_{1}-z_{2}\right)^{k+1}}\right| \leqq M_{k}
$$

for all $z_{1}$ and $z_{2}$ on $C, z_{1} \neq z_{2}$.

The Lebesgue measure in the theorem means measure on the $\theta$-line after the transformation $\phi^{-1}: z \rightarrow \theta$. write

To prove this lemma, we let $z_{1}=\phi\left(\alpha_{1}\right), z_{2}=\phi\left(\alpha_{2}\right), t=\phi(\theta)$, and

$$
\begin{aligned}
& \frac{J_{k}\left(z_{1}, z_{2}\right)}{\left(z_{1}-z_{2}\right)^{k+1}} \\
& \quad=\left[\frac{e^{i \alpha_{1}}-e^{i \alpha_{2}}}{\phi\left(\alpha_{1}\right)-\phi\left(\alpha_{2}\right)}\right]^{k+1} \frac{\int_{\alpha_{2}}^{\alpha_{1}}\left(e^{i \theta}-e^{i \alpha_{2}}\right)^{k} g\left(e^{i \theta}\right) i e^{i \theta} d \theta}{\left(e^{i \alpha_{1}}-e^{i \alpha_{2}}\right)^{k+1}},
\end{aligned}
$$

where

$$
g\left(e^{i \theta}\right) i e^{i \theta}=G(\phi(\theta))\left[\frac{\phi(\theta)-\phi\left(\alpha_{2}\right)}{e^{i \theta}-e^{i \alpha_{2}}}\right]^{k} \psi(\theta) .
$$

By Lemma 3.1, the quantities in the square brackets in (3.1) and (3.2) 
are both uniformly bounded in modulus for all $\theta, \alpha_{1}$, and $\alpha_{2}, \theta \neq \alpha_{2}, \alpha_{1} \neq \alpha_{2}$. For any fixed $\alpha_{2}, g\left(e^{i \theta}\right)$ as given by (3.2) is integrable, and its modulus is uniformly bounded for all $\theta$ and $\alpha_{2}$. The integral in (3.1), considered as an integral over an arc of the unit circle, is independent of the path of integration. Thus the hypotheses of Lemma 2.2 are satisfied by $g$ as given by (3.2). The truth of Lemma 3.3 now follows immediately.

Theorem 3.1 can now be proved by the use of Lemmas 3.2 and 3.3 in the same way that Theorem 2.1 was proved. The hypotheses on $f$ and $C$ imply that $f^{n-1}\left(e^{i \theta}\right)$ is an absolutely continuous function of $e^{i \theta}$ and of $\theta$, and that its derivative with respect to $\phi(\theta)$, where it exists, is uniformly bounded in modulus. The same is true for its derivative with respect to $\theta$. (These facts follow from the existence of numbers $\lambda_{1}$ and $\lambda_{2}$ such that with $z=e^{i \theta}, t=e^{i \alpha}$,

$$
\left|f^{n-1}(z)-f^{n-1}(t)\right| \leqq \lambda|\phi(\theta)-\phi(\alpha)| \leqq \lambda_{1}\left|e^{i \theta}-e^{i \alpha}\right| \leqq \lambda_{2}|\theta-\alpha| .
$$

(Here we used Lemma 3.1 in passing from the second member to the third member of the chain.) The functions $f, f^{1}, \cdots, f^{n-2}$ are also absolutely continuous and have uniformly bounded derivatives.

We can now re-establish the recursion formulas (2.10), which look exactly the same as before and so will not be repeated here. The integrals in (2.10) are of course now complex line integrals over $C$. Thereafter by back-substitution, using Lemma 3.3 at each stage, we establish the existence of the bound for $\left|d_{n}\right|$.

The analogous generalization of Theorem 2.2 is also valid. The proper definition of $d_{n}$ for confluent points is again given by (2.11). It is worth noting that what gives simplicity to our results and minimizes the restrictions on $C$ is the complex-variable type of definition which we are using for derivatives of functions given on $C$.

4. Some asymptotic properties of divided differences formed on a Jordan curve. In this section we shall be considering an infinite sequence of divided differences

$$
d_{1}\left(f \mid z_{11}, z_{12}\right), d_{2}\left(f z_{21}, z_{22}, z_{23}\right), \cdots, d_{n}\left(f \mid z_{n 1}, z_{n 2}, \cdots, z_{n}, z_{u+1}\right), \cdots,
$$

formed for a function $f$ given on a Jordan curve $C$ in the $z$-plane. Do there exist sequences of point sets $S_{n+1}=\left\{z_{n 1}, z_{n 2}, \cdots, z_{n},{ }_{n+1}\right\}, n=1,2, \cdots$ such that $\lim _{n \rightarrow \infty} d_{n}$ exists for all functions $f$ belonging to an interestingly wide class; and if so, what is this limit?

Let $D$ be the region interior to the curve $C$, let $K$ be the unlimited region exterior to $C$, and let $\bar{D}$ be $D \cup C$. There exists an analytic function

$$
z=\chi(w)=c w+c_{0}+\frac{c_{1}}{w}+\frac{c_{2}}{w^{2}}+\cdots, c>0
$$


univalent for $|w|>1$, which maps $|w|>1$ conformally onto $K$ so that the points at infinity in the $z$-plane and $w$-plane correspond. According to the Osgood-Taylor-Carathéodory Theorem, $\chi(w)$ can be extended in a continuous and one-to-one manner onto $|w|=1$, and $\chi\left(e^{i \theta}\right)=\phi(\theta)$ then gives a parametric equation for $C$ of the type considered above in $\S 3$. The number $c$ is called the transfinite diameter (Robin's constant, capacity) of $C$.

If a function $f$ is analytic on $C$, it is also analytic in a region (perhaps multiply connected) which contains $C$ in its interior. Let $w=R e^{i \theta}$ in (4.1). There is a largest value of $R$, say $\rho>1$, such that $f$ is analytic at every point of the intersection of $C \cup K$ with the region interior to the Jordan curve $C_{R}: z=\chi\left(R e^{i \theta}\right), 0 \leqq \theta \leqq 2 \pi$. (See [9, p. 79].) A curve such as $C_{R}$ is called a level curve of the map given by (4.1).

With $z_{n k}=\chi\left(e^{i \theta_{n k}}\right), 0 \leqq \theta_{n k}<2 \pi$, let $N_{n}(\theta)$ be the number of elements of the set $\left\{\theta_{n 1}, \theta_{n 2}, \cdots, \theta_{n, n+1}\right\}$ falling into the closed interval $[0, \theta]$. The numbers $\theta_{n k}, k=1, \cdots, n+1, n=1,2, \cdots$ are said to be equidistributed on $[0,2 \pi]$ if $\lim _{n \rightarrow \infty} N_{n}(\theta) /(n+1)=\theta / 2 \pi$; and when this happens, the corresponding sequence of point sets $S_{n+1}, n=1,2, \cdots$, is said to be equidistributed on $C$.

Our first result is as follows:

Theorem 4.1. Let $f$ be analytic on $\bar{D}$ and let the sequence $\left\{S_{n+1}\right\}$ be equidistributed on $C$. Let $\rho$ be the largest value of $|w|$ in the map (4.1) such that $f$ is analytic interior to the level curve $C_{|w|}$. Then for any $R, 1<R<\rho$, there exists a constant $M$ depending on $f, R$, and $C$, but not on $n$, such that

$$
\left|d_{n}\left(f \mid z_{n 1}, \cdots, z_{n},,_{n+1}\right)\right| \leqq \frac{M}{(c R)^{n+1}} .
$$

Thus $\lim _{n \rightarrow \infty} c^{n+1} d_{n}=0$.

To prove this, we use the formula $[5, p .11]$

$$
d_{n}=\frac{1}{2 \pi i} \int_{\sigma_{R}} \frac{f(t)}{\omega_{n+1}(t)} d t, \quad 0<R<\rho,
$$

where $\omega_{n+1}(z)=\left(z-z_{n 1}\right)\left(z-z_{n 2}\right) \cdots\left(z-z_{n, n+1}\right)$. This formula can be used to complete the definition of $d_{n}$ by continuity for the case of confluent points $z_{n k}$. We then refer to a classical result of L. Fejér [4], [9, pp. $167 \mathrm{ff}]$ : If $\left\{S_{n+1}\right\}$ is equidistributed on a Jordan curve $C$, then

$$
\lim _{n \rightarrow \infty}\left|\omega_{n+1}(z)\right|^{1 /(n+1)}=c|w|, z=\chi(w),
$$

uniformly for $z$ on any closed subset of $K$. This implies that if $z$ lies on $C_{R}$ and $R_{1}$ is such that $1<R_{1}<R$, then for all $n$ sufficiently large, 
LIMITS AND BOUNDS FOR DIVIDED DIFFERENCES ON A JORDAN CURVE 1231

$$
\frac{c^{n+1}}{\left|\omega_{n+1}(z)\right|} \leqq \frac{1}{R_{1}^{n+1}}
$$

Letting $M_{R}$ be the maximum of $|f(z)|$ on $C_{R}$, and $L_{R}$ be the length of $C_{R}$, we appraise (4.2) as follows:

$$
c^{n+1} \mid d_{n} \leqq \frac{M_{R} L_{R}}{2 \pi R_{1}^{n+1}}
$$

and the theorem follows from this.

THEOREM 4.2. Let $C$ be rectifiable, let $f$ be analytic on $C$, and let $S_{n+1}$ be the transform under (4.1) of $n+1$ distinct points equally spaced on $|w|=1$. Then

$$
\lim _{n \rightarrow \infty} c^{n+1} d_{n}=\frac{1}{2 \pi i} \int_{0} f(t) d t
$$

This is consistent with Theorem 4.1 , because the sequence $\left\{S_{n+1}\right\}$ in Theorem 4.2 is equidistributed and the integral in (4.4) would be zero if $f$ were analytic on $\bar{D}$.

To prove the theorem we use a generalization of (4.2),

$$
d_{n}=\frac{1}{2 \pi i}\left(\int_{o_{R}}+\int_{\sigma^{\prime}}\right) \frac{f(t)}{\omega_{n+1}(t)} d t,
$$

which is easily established by the calculus of residues. Here $C_{R}, R>1$, is a level curve of (4.1) and $C^{\prime}$ is a suitably chosen rectifiable curve lying in $D$. The curves $C_{R}$ and $C^{\prime}$ are chosen so that $f$ is analytic on the closed annular region bounded by $C_{R}$ and $C^{\prime}$. Integration on $C^{\prime}$ is in the opposite sense to that on $C_{R}$.

The appraisal given by (4.3) is valid for the first integral in (4.5), and it shows that this integral vanishes in the limit. The following result of the author [2] is available for the second integral: From the hypotheses of Theorem 4.2 on $C$ and $S_{n+1}$ it follows that

$$
\lim _{n \rightarrow \infty} \omega_{n+1}(z) / c^{n+1}=-1
$$

uniformly for $z$ on any closed subset of $D$. This implies that

$$
\begin{aligned}
\lim _{n \rightarrow \infty} & \frac{1}{2 \pi i} \int_{\sigma^{\prime}} \frac{c^{n+1} f(t)}{\omega_{n+1}(t)} d t \\
& =\frac{1}{2 \pi i} \int_{\sigma^{\prime}} \lim _{n \rightarrow \infty} \frac{c^{n+1} f(t)}{\omega_{n+1}(t)} d t \\
& =-\frac{1}{2 \pi i} \int_{\sigma^{\prime}} f(t) d t=\frac{1}{2 \pi i} \int_{\sigma} f(t) d t,
\end{aligned}
$$


which completes the proof.

Generalizations of the above theorems to the case in which $D$ is replaced by a finite number of mutually exterior Jordan regions can be developed by the methods to be found in Walsh's book [9, Chap. VII].

The results from which the above two theorems are derived were originally established in studying the convergence of sequences of polynomials found by interpolation to the function $f$ on $C$. Let $L_{n+1}(z)=$ $L_{n+1}\left(f ; z \mid S_{n+1}\right)$ be the (unique) polynomial in $z$ of degree at most $n$ which is determined by the condition that it shall coincide with $f(z)$ at each of the points $S_{n+1}$, assumed to be distinct. Then from the standard formula

$$
L_{n+1}(\alpha)=\omega_{n+1}(\alpha) \sum_{k=1}^{n+1} \frac{f\left(z_{n k}\right)}{\omega_{n+1}^{\prime}\left(z_{n k}\right)\left(\alpha-z_{n k}\right)}
$$

it is seen by comparison with (1.1) that

$$
L_{n+1}\left(g ; \alpha \mid S_{n+1}\right)=\omega_{n+1}(\alpha) d_{n}\left(f \mid z_{n 1}, \cdots, z_{n, n+1}\right)
$$

where $f(z)=g(z) /(\alpha-z)$. The following result of the author [2], [3] is relevant: Let the curve $C$ be such that $\chi^{\prime}(w)$ is nonvanishing and of bounded variation for $|w|=1$. Let $g$ be bounded and integrable in the sense of Riemann on $C$. Let the points $S_{n+1}$ be the transforms under (4.1) of distinct points equally spaced on the unit circle. Then

$$
\lim _{n \rightarrow \infty} L_{n+1}\left(g ; \alpha \mid S_{n}\right)=\frac{1}{2 \pi i} \int_{o} \frac{g(t)}{t-\alpha} d t
$$

uniformly for $\alpha$ on any closed subset of $D$.

We now write (4.6) in the form

$$
c^{n+1} d_{n}=\left[-\frac{c^{n+1}}{\omega_{n+1}(\alpha)}\right]\left[-L_{n+1}\left(g ; \alpha \mid S_{n+1}\right)\right] .
$$

If $\alpha$ is a fixed point of $D$ and $f$ is bounded and Riemann integrable on $C$, then so is $g$ and conversely. We recall that $-\omega_{n+1}(\alpha) / c^{n+1}$ tends to unity at each point of $D$ as $n$ becomes infinite. It follows from these facts that the limiting value of (4.7) is

$$
\lim _{n \rightarrow \infty} c^{n+1} d_{n}=\frac{-1}{2 \pi i} \int_{o} \frac{g(t)}{t-\alpha} d t=\frac{1}{2 \pi i} \int_{o} f(t) d t .
$$

We summarize formally:

THEOREM 4.3. If the points $S_{n+1}$ are transforms under (4.1) of distinct points equally spaced on the unit circle, and if $C$ is such that $\chi^{\prime}$ is nonvanishing and of bounded variation for $|w|=1$, and if $f$ is 
LIMITS AND BOUNDS FOR DIVIDED DIFFERENCES ON A JORDAN CURVE 1233

bounded and integrable in the sense of Riemann on $C$, then

$$
\begin{gathered}
\lim _{n \rightarrow \infty} c^{n+1} d_{n}=\frac{1}{2 \pi i} \int_{o} f(t) d t . \\
\text { REFERENCES }
\end{gathered}
$$

1. J. H. Curtiss, "Interpolation in equidistributed points on the unit circle", to appear in the Pacific Journal of Mathematics.

2. - Riemann sums and the fundamental polynomials of Lagrange interpolation" Duke Math. 8 (1941), 634-646.

3. _ Interpolation in regularly distributed points, Trans. Amer. Math. Soc., 38 (1935), 458-473.

4. L. Fejér, "Interpolation und konforme Abbildung, Gottinger Nachrichten, (1918), 319331.

5. Milne-Thomson, L. M., The Calculus of Finite Differences, MacMillan and Co., London, 1933.

6. W. E. Sewell, Degree of Approximation by Polynomials in the Complex Domain, Princeton University Press, Princeton, 1942.

7. J. F. Steffensen, Interpolation, Williams and Wilkins, Baltimore, 1927.

8. E. C. Titchmarsh, Theory of Functions, Oxford University Press, Oxford, 1932.

9. J. L. Walsh, Interpolation and Approximation by Rational Functions in the Complex Domain, Second edition, Amer. Math. Soc., Providence, 1956.

UNIVERSITY OF MIAMI 
